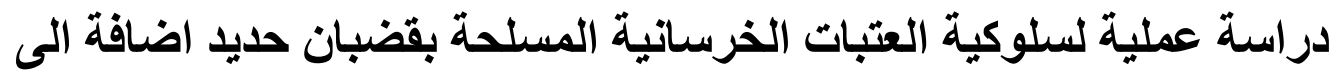 الالياف القولاذية تحت تاثير الاحمال التكرارية التية المبلة
}

\author{
سميز سعدي شماس \\ منى مبارك عبدالله \\ د. بيار جعفر السليفاني \\ م.مساعد، قسم الهندسة المدنية \\ م.مساعد، قسم الهندسة المدنية \\ استاذ، قسم الهندسة المدنية
}

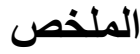

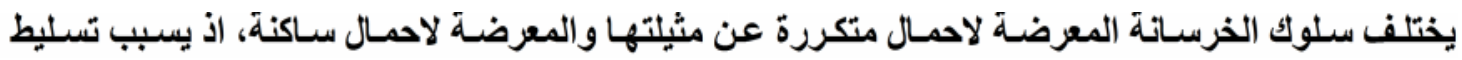

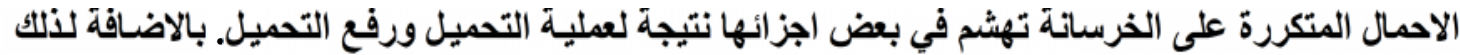

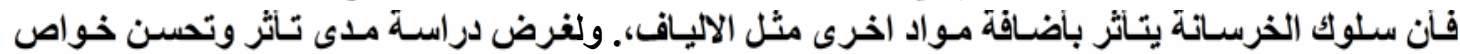

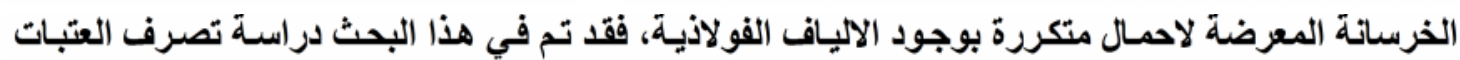

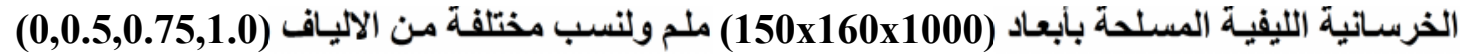

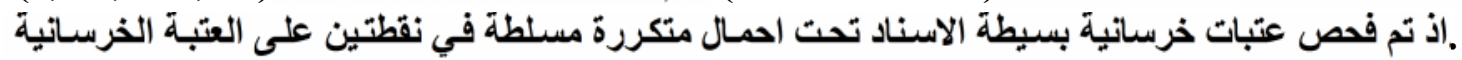

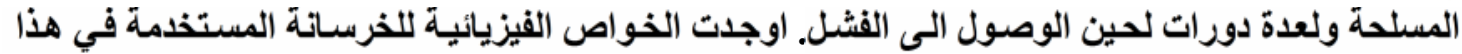

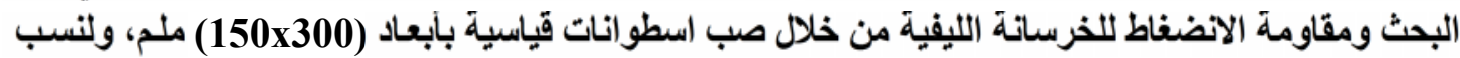

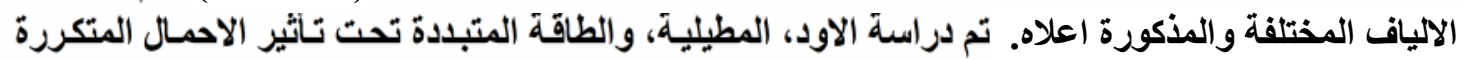

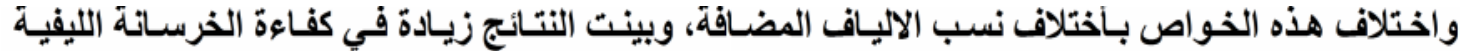

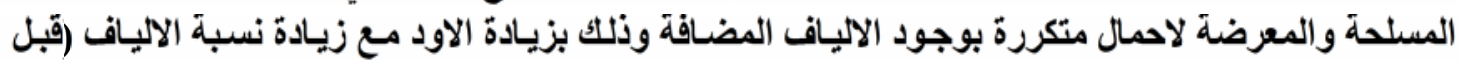
الفثل) ، وكنلك زيادة المطيلية وزيادة الطاقة المتبدة بوجود الالياف نتيجة عمليات التحميل ورفع التحميل.

\section{Experimental Study of the Behavior of Fiber Reinforced Concrete Beams under Repeated Loads}

\section{Dr.Bayar J. Al - Sulayfani}

Dept.civil engineering
Muna M

\section{.Abdullah}

Dept.civil engineering
Samier S. Shmas

Dept.civil engineering

\begin{abstract}
:
Concrete behavior subjected to repeated loads is differs than the one subjected to static loads. Repeated loads caused crushing in some part of concrete due to loading and unloading process. Concrete behavior under static loads is affected by adding steel fibers, which improved many properties especially concrete tensile strength. These improvements are studied in this research under the effect of repeated loads, by testing simply supported fibrous reinforced concrete beams with dimensions $(1000 \times 150 \times 160) \mathrm{mm}$, reinforced with different
\end{abstract}


percentages of steel fibers $(0.0,0.5,0.75$, and $1.0 \%)$. Repeated loads were applied to the beams through two points and for many cycles up to failure. The physical properties and compressive strength of the concrete used was found by casting standard cylinders $(150 \times 300) \mathrm{mm}$ for the different percentages of fibers. All the results show improvements in beams behavior due to fiber addition to the concrete under repeated loads, by increasing the deflection, strain, ductility and energy dissipation due to increasing of added fibers percentages.

Keywords : Repeated load, Reinforced Concrete, Beams, Steel fiber

\footnotetext{
قبل في 2009/10/22

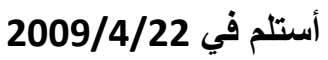

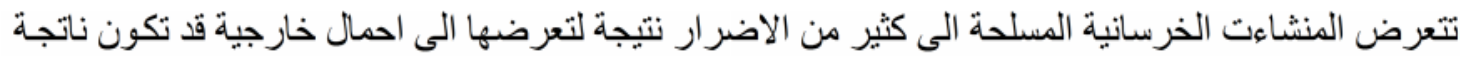

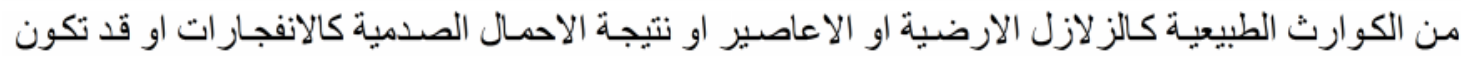

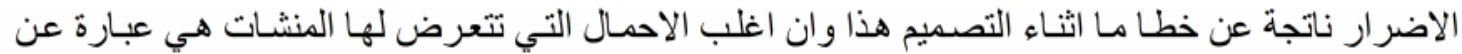

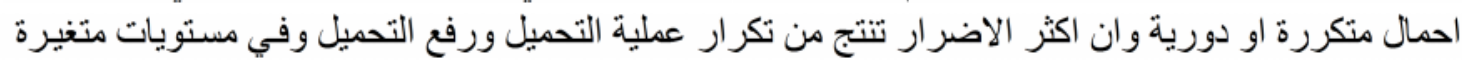

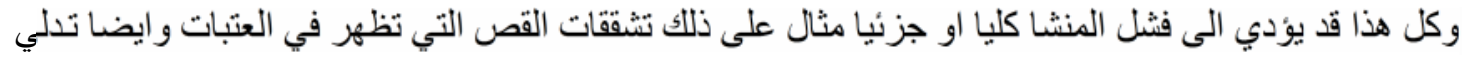

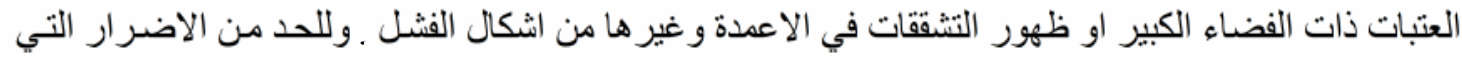

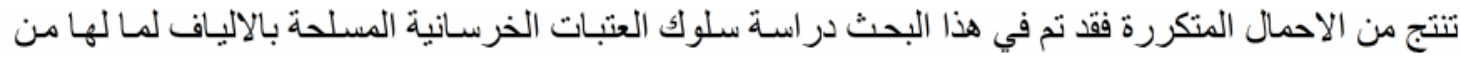

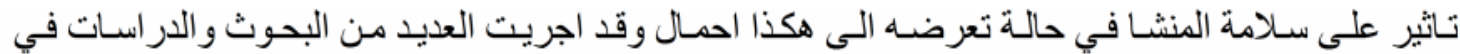

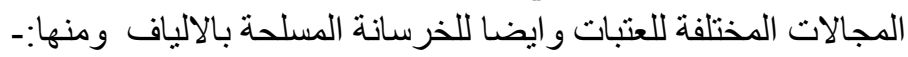

قيام الباحث Hsu Sinha و1 [1] في عام 1964 بـاجر اء تجارب عملية على مجمو عـة من العتبات ذات مقطع

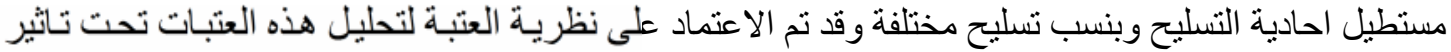

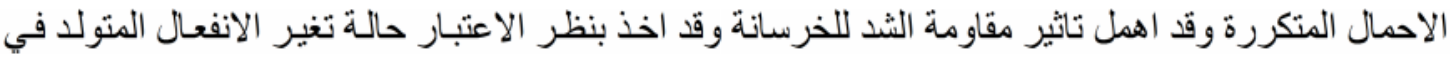

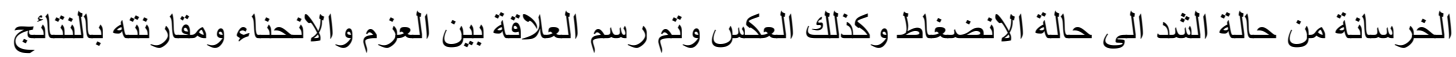

العملية.

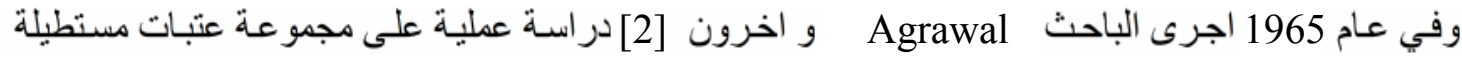

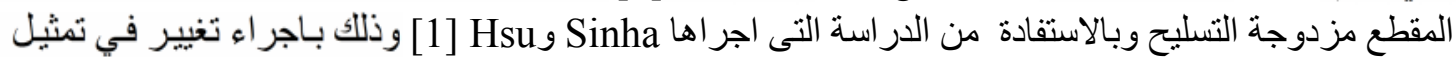

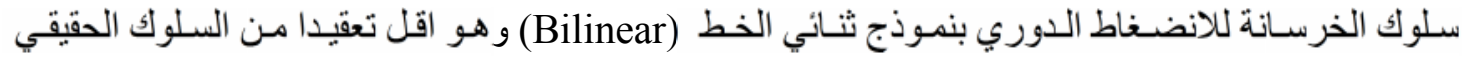
للخرسانة لهذا النوع من الاحمال وقد اظهر هذا النموذج نو افقا جيدا مع الفحوصات الغطونية.

قيام الباحث Al-Sulayfani [3] عام (1986) بأجر اء فحوصـات عملية على عتبات خرسانية مسلحة بسيطة الاسناد ، معرضة اللى احمال دورية ومسلحة بنسب مختلفة من حديد التسليح .تم رسم منحنيات علات علمة (العزم-
} 
الانحناء ) و (الحمل-الاز احة) في المقاطع الحرجة للعتبات التي تم فحصها.وقورنت مع نتائج فحوصـات عملية ونظرية اخرى و اظهرت تطابقاً جيدأ.

وفي عام 2001 قام الباحث Depongpan Thammanoon [4] بدر اسة عملية على عتبات خرسانية مسلحة

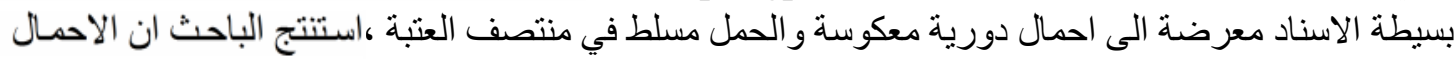

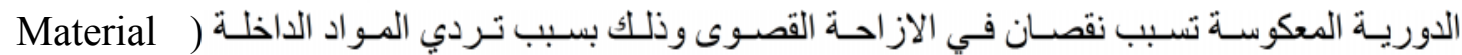
(Deterioration المعرضة للاحمال المعكوسة يقل تقريبأ بمقدار (10 40) من العتبات المعرضة للاحمال التز ايدية ( Monotonic

. (Loads

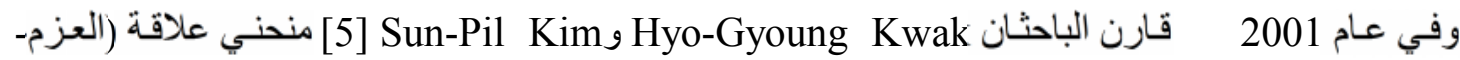

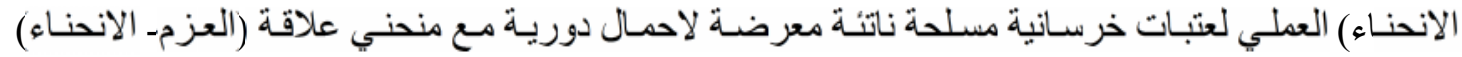

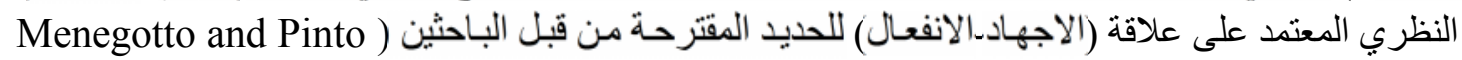

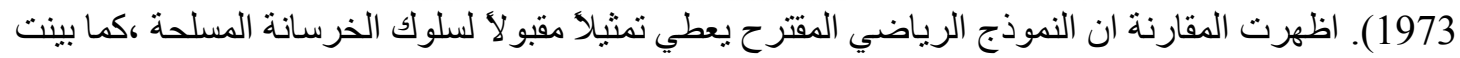

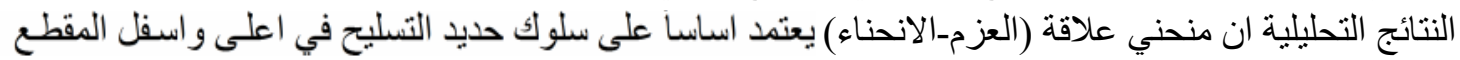
الخرساني . (النتائ.

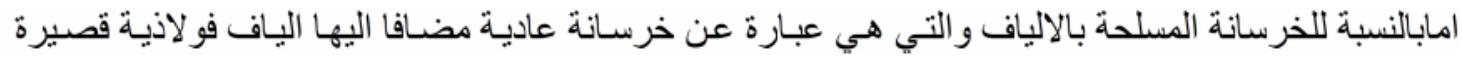

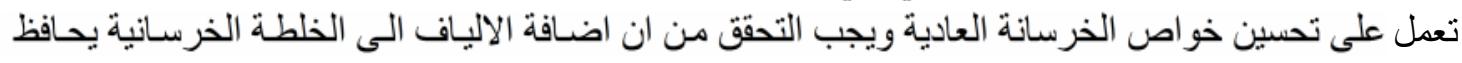

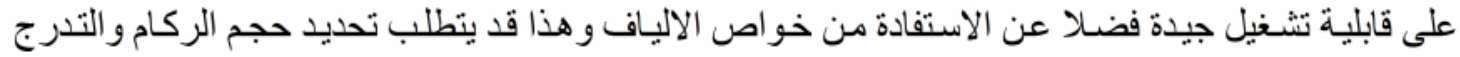

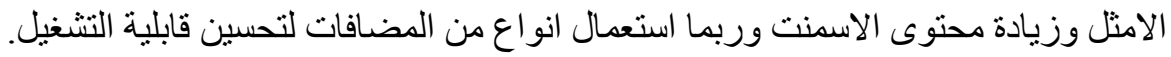

تصنع الالياف الفو لاذية باشكال مختلفة ،ويكون مقطعها دائريا او نصف دائري او مستطيلا او متغير اوقد تكون

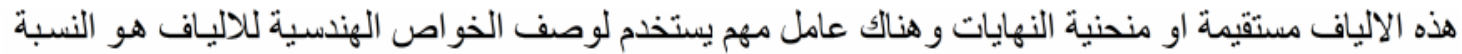

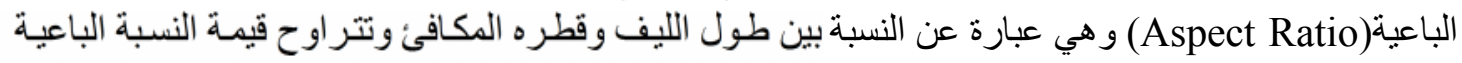
بين (150-30). ومن الدراسات في هذا المجال:

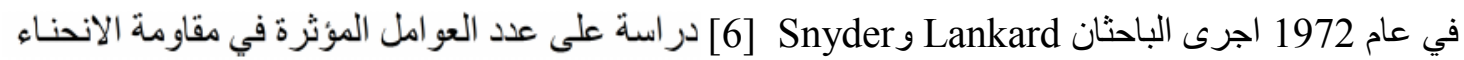

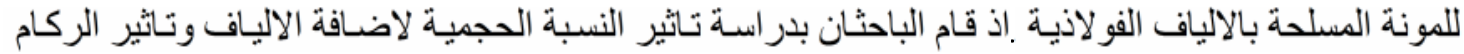
وتاثير المسافة بين الالياف في مقاومة الانحناء للمونة المسلحة بالالياف. لإنة

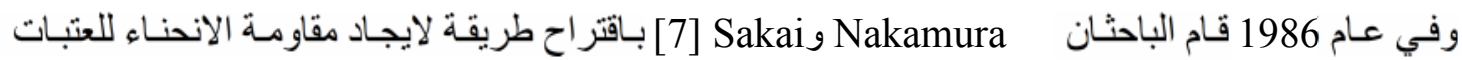

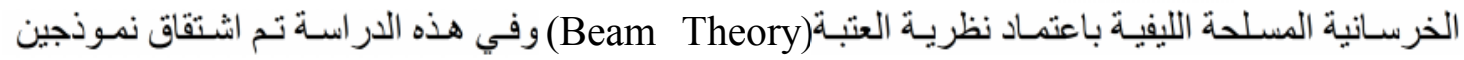
رياضيين لمنحني الاجهاد_الانفعال للخرسانة الليفية تحت تانثير الثد باعتماد نو عين مختلفين من الالياف الفو لاذية.

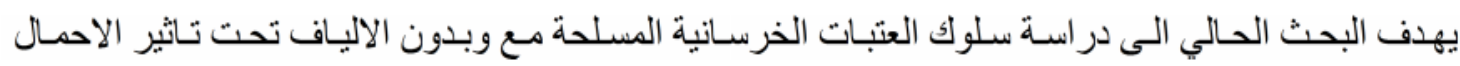
المتكررة ومعرفة تاثير اختلاف نسب الالياف على مقدار الاود الناتج من تسليط هذا النوع من الاحمال.

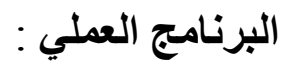

يتضمن البرنامج العملي تعريف بـالمو اد المستخدمة لاعداد الخلطة الخرسـانية المعتمدة في البحث فضـلا عن

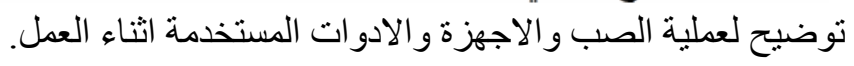


الخلطة الخرسانية: ان الخرسانة هي عبارة عن خليط من اربعة عناصر اساسية وهي كل من الاسمنت و الرمل

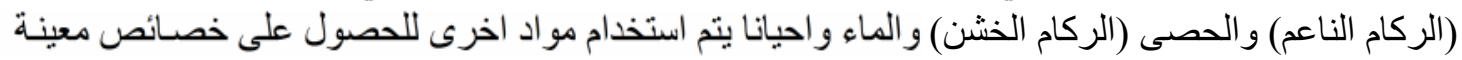

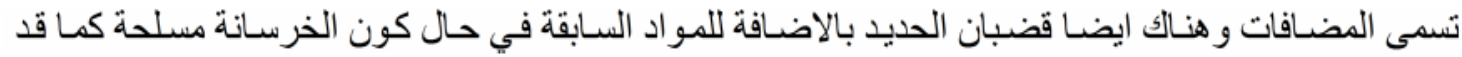

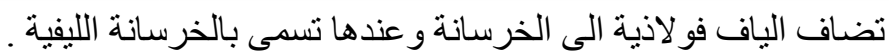

1 ـ الاسمنت :في هذا البحث استخدم اسمنت عر اقي محلي تتم صناعته في محافظة نينوى من نوع بـادوش وهذا الاسمنت من نوع بورتلاند الاعنيادي.

זـ الماء: تم استخدام مـاء الثرب الاعتيادي لمدينـة الموصل في الخلطة الخرسـانية ومعالجـة النمـاذج وهو مـاء

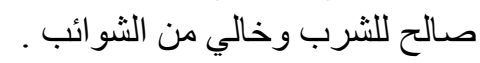

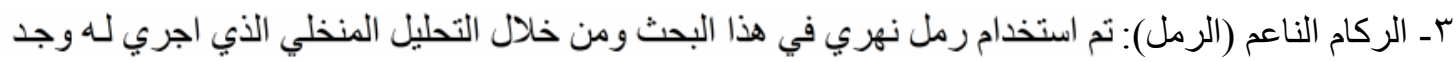
انه ضمن الحد العام للمو اصفات البريطانية (BS882:1983) [8] و هو من نوع الرمل فت فتوسط النعومة . عـ الركام الخشن ( الحصى): الحصى المستخدم في البحث هو حصى نهزي و المسمى محليا (البحص)

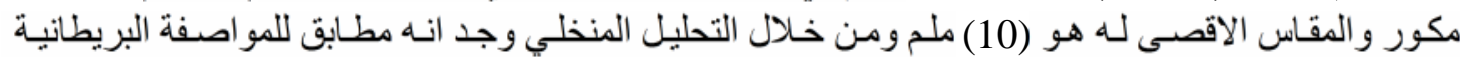

.[8] (BS882:1983)

-نسب الخلطة الخرسانية: للحصول على الخرسانة بالمقاومة المطلوبة تم صب عدة خلطات خرسانية تجريبية

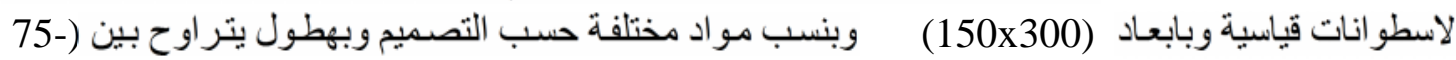
100) ملم اختيرت الخلطة الخرسانية ذات النسب الوزنية)

- حديد التسليح: تم استخدام قضبان حديد التسليح الرئيسي بق (12)

ـالالياف الفو لاذية:تم استخدام الياف فو لاذية وبنسب مختلفة وكما يلي \%(0,0.5,0.75,1.0). و النسبة الباعية

ويمكن ملاحظة المواد المستخدمة في عملية الصب كما في النكل( )

\section{طريقة صب النماذج الخرسانية:}

تبدا عملية الصب بعد تنظيف قو الب الصب جيدا ثم تدهن القو الب من الداخل بطبقة خفيفة من الزيت لتسهيل

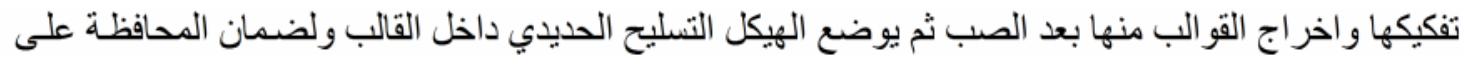

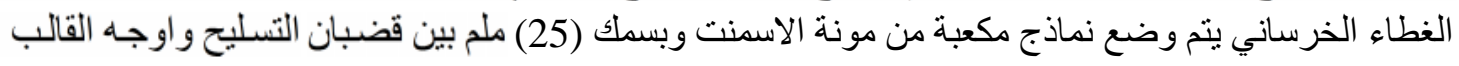




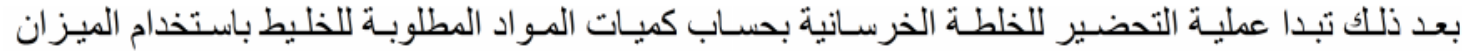

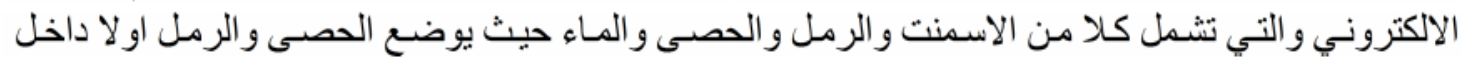

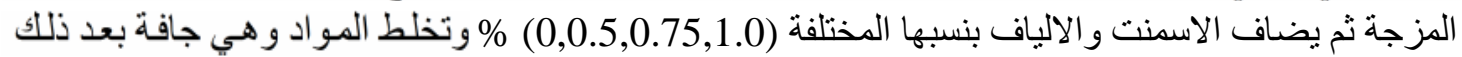

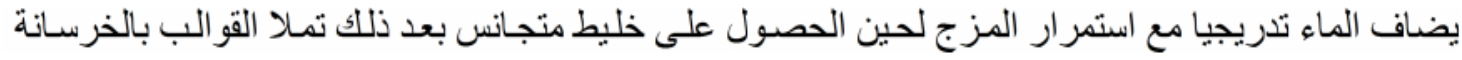

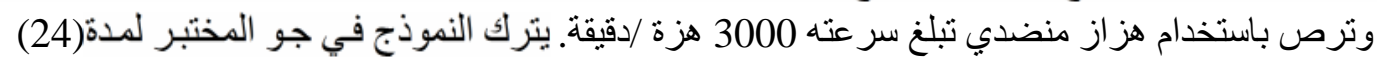

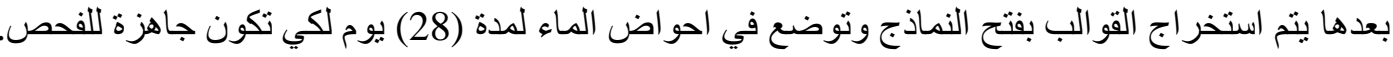

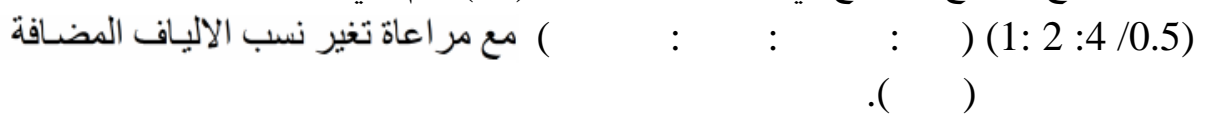
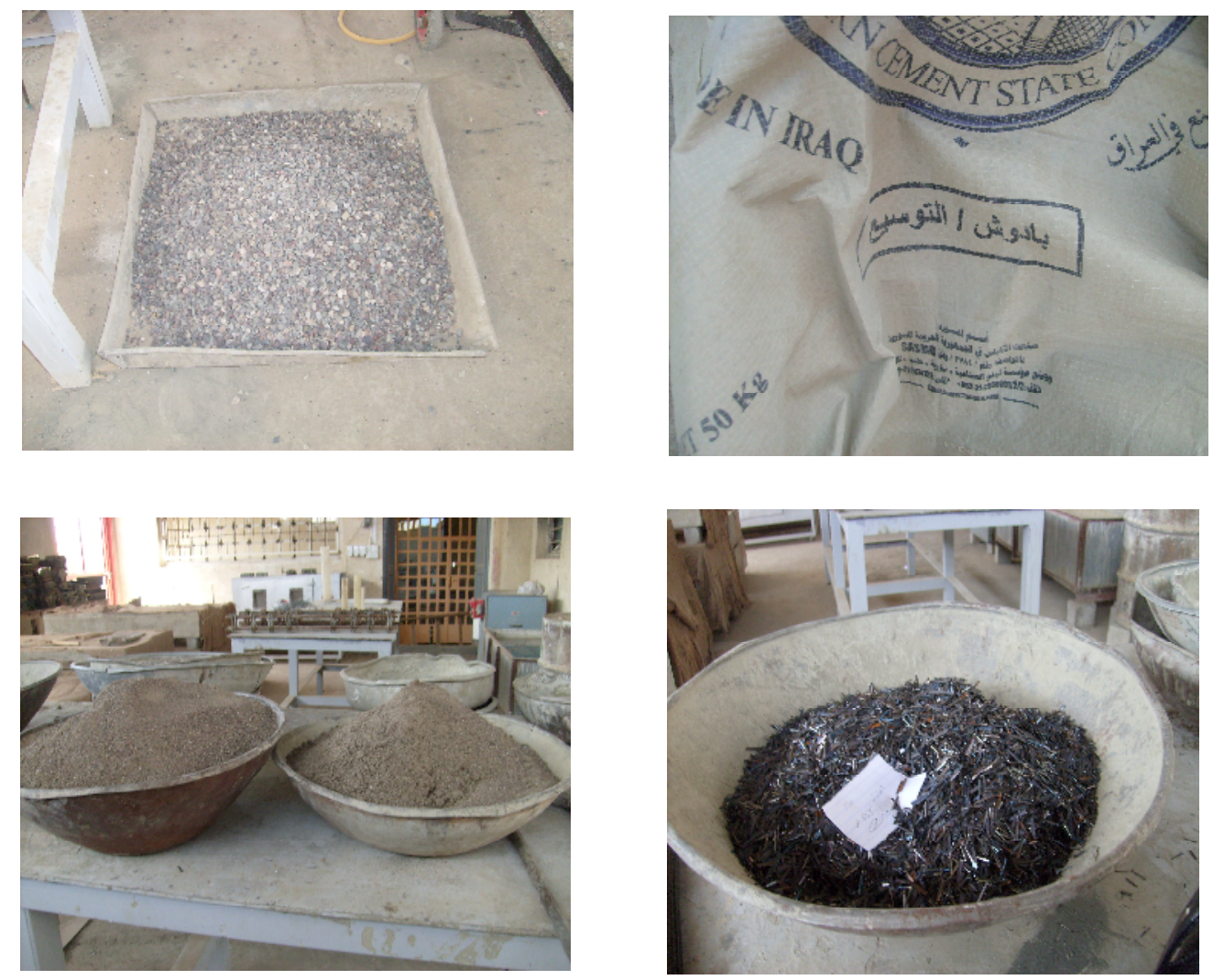

شكل رقم (1) المواد المستخدمة في عملية الصب 

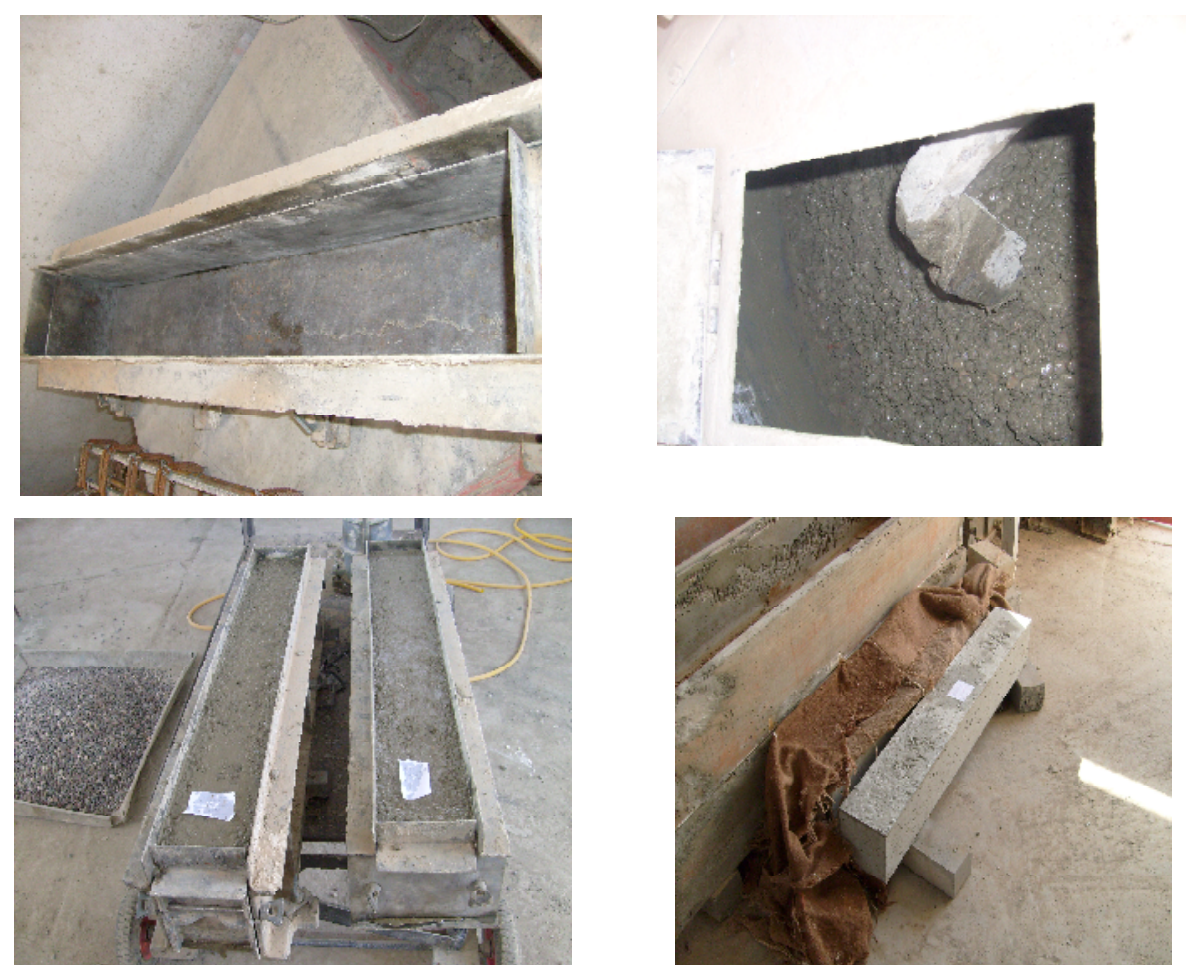

شكل (2) خطوات عملية الصب و القو الب المستخدمة 


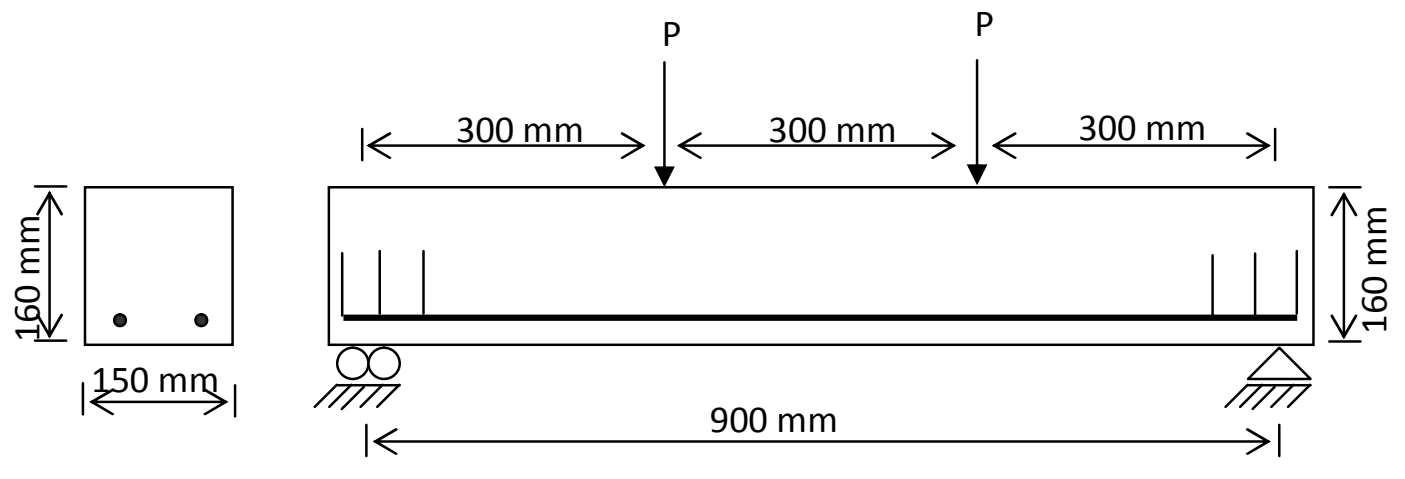

شكل(3) مخطط يوضح ابعاد النموذج المستخدم مع اماكن تسليط الحمل 


\section{الاجهزة المستخدمة في الفحص:}

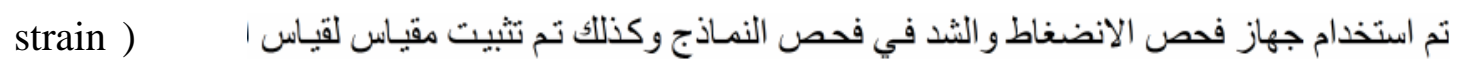

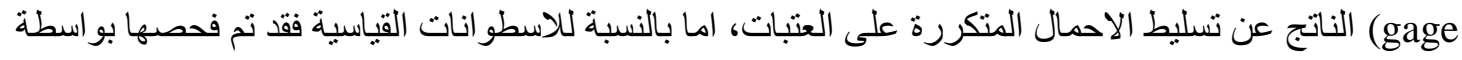

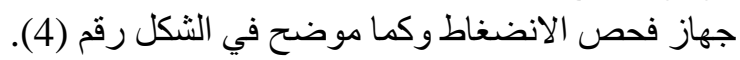
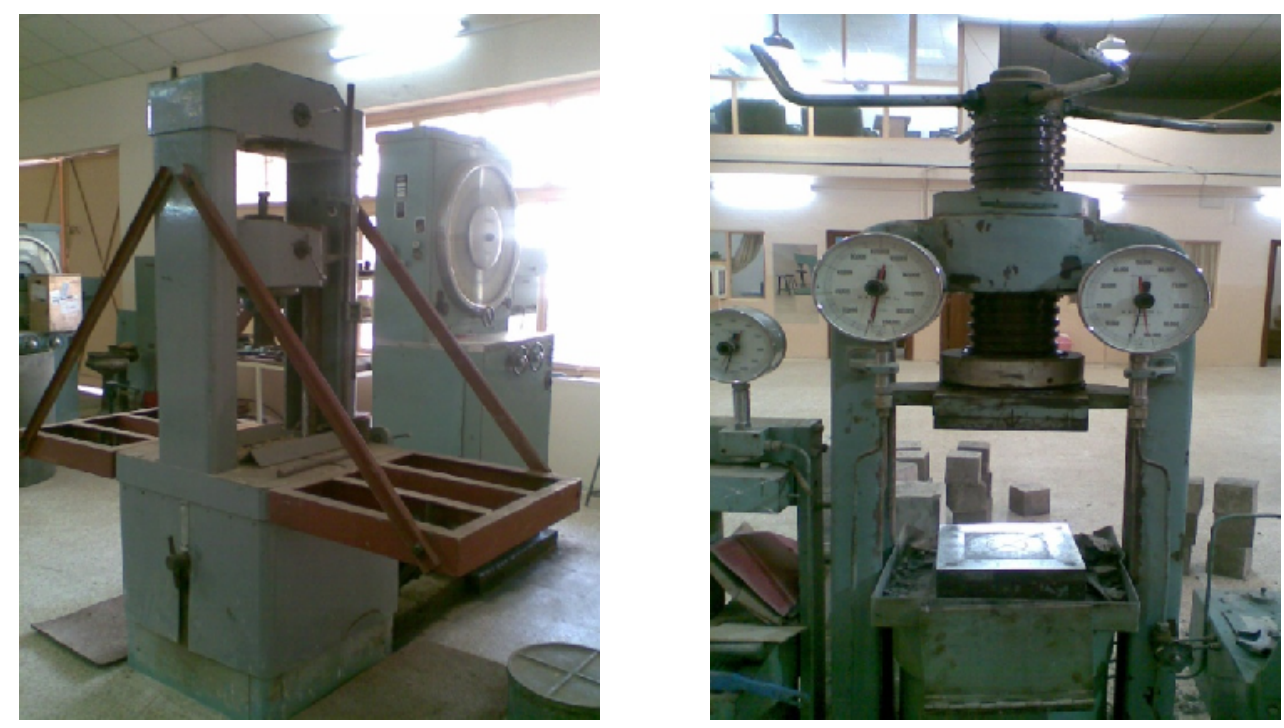

شكل (4) الاجهزة المستخدمة في الفحص 
- فحص اسطو انات قياسية بابعـاد( ) 300 150) ملم من نفس الخلطة التي تم صب النمـاذج الرئيسية منها :( )

(1) يبين مقاومة الانضغاط للاسطو انات القياسية مع النسبة المئوية للالياف

\begin{tabular}{|c|c|c|}
\hline مقاومة الانضغاط (ميكاباسكال) & حمل الفشل (طن) & نسبة الالياف \\
\hline . & 65.5 & $0 \%$ \\
\hline . & 70.5 & $0.5 \%$ \\
\hline & 75.5 & $0.75 \%$ \\
\hline
\end{tabular}

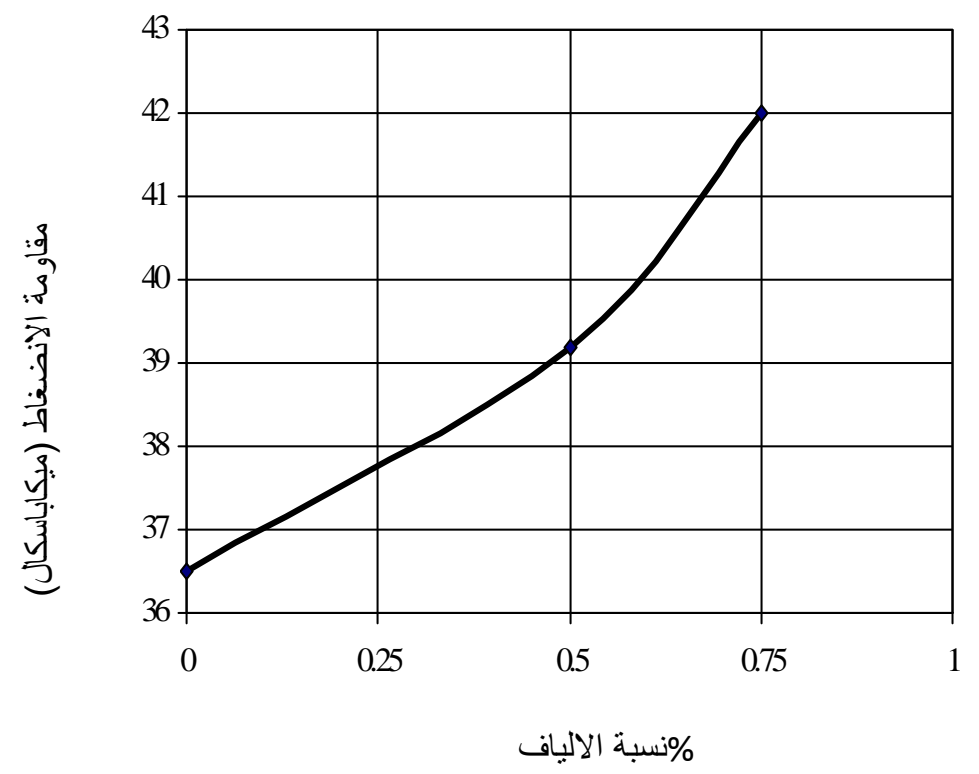

شكل(5) العلاقة بين مقاومة الانضغاط للاسطو انات القياسية مع النسبة المئوية للالياف 
1.

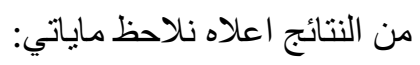

· زيادة مقاومة الانضغاط بالنسبة الى نسب الالياف التي تعزى الى وجود الالياف داخل

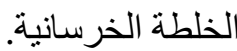

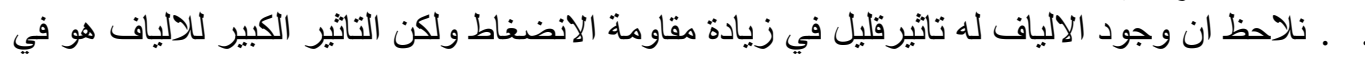

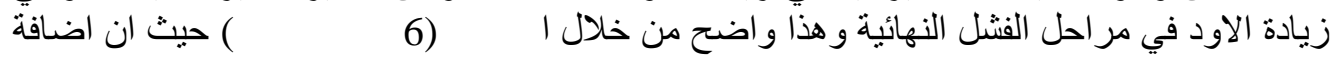

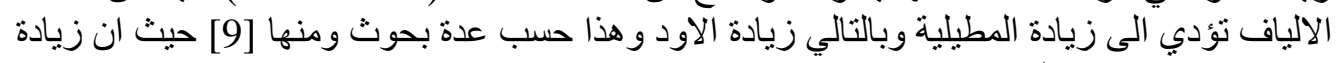

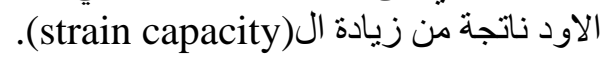

ـ تم فحص عتبات خرسانية مسلحة بابعاد(160x 150x1000) ملم و الحصول على النتائج التالية:
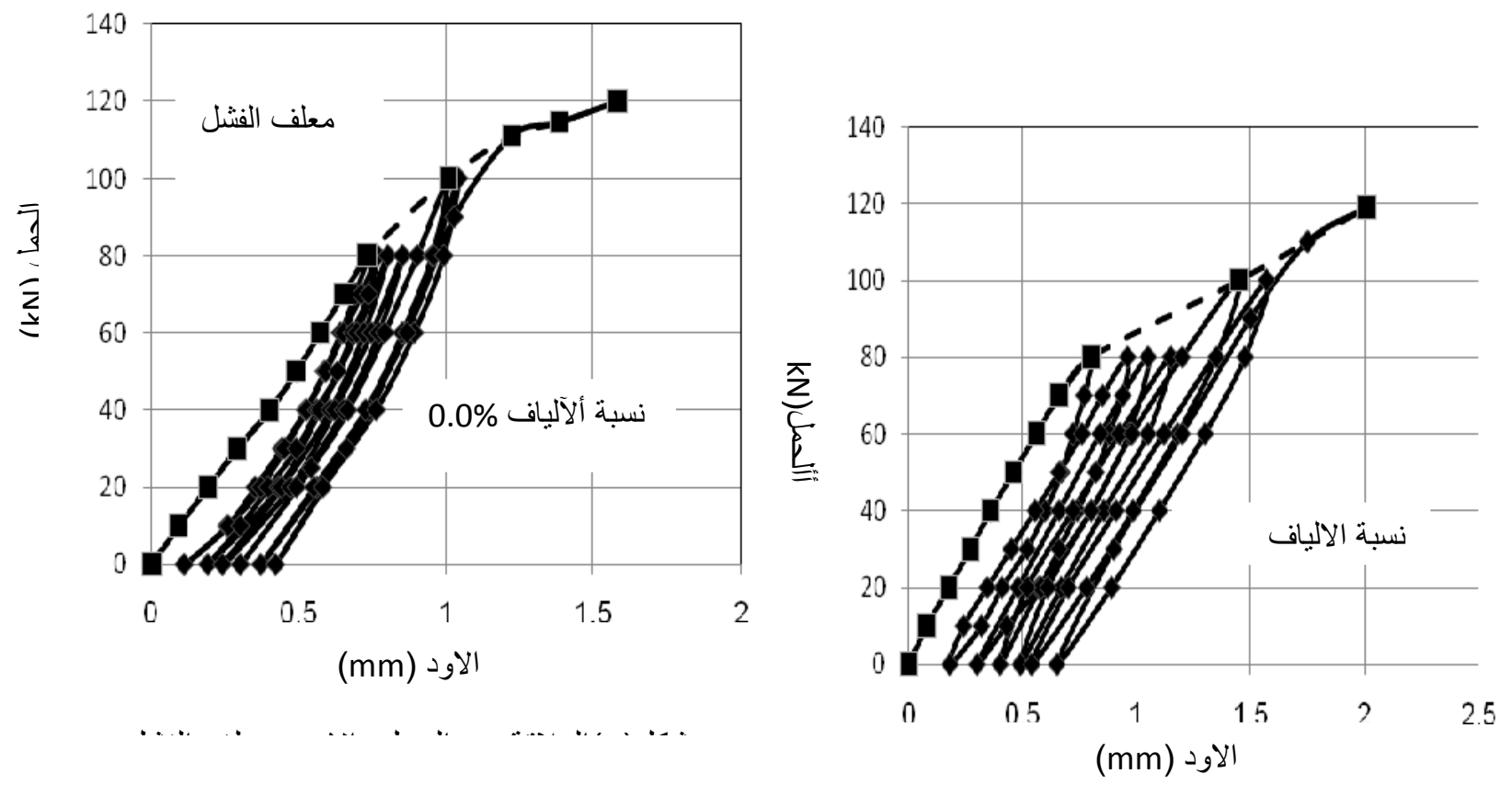

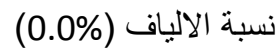




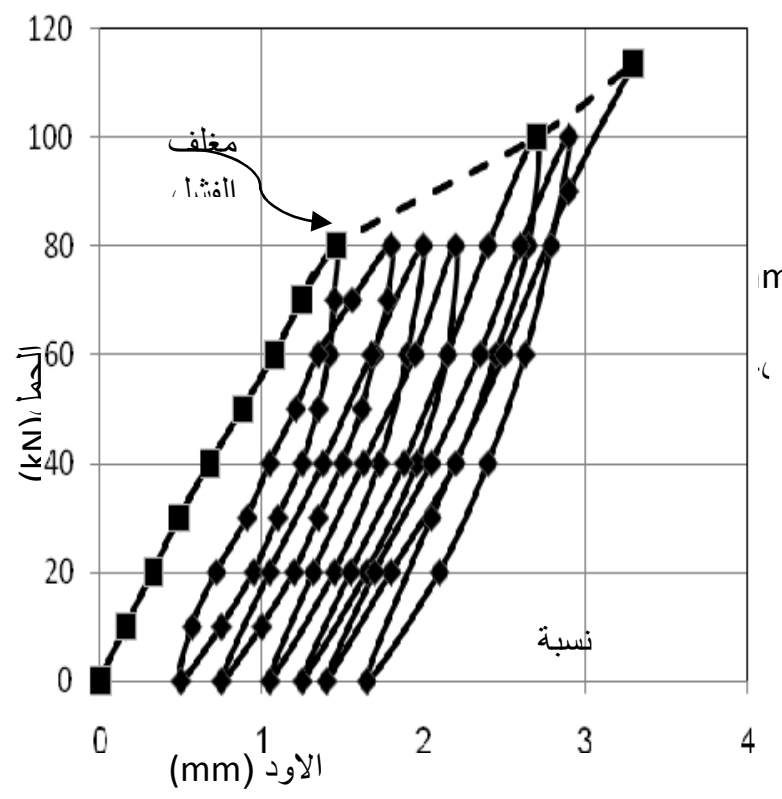

شكا (م)/العلاقة سن, الحما ، - الاهد دم مغلف الفشا ،

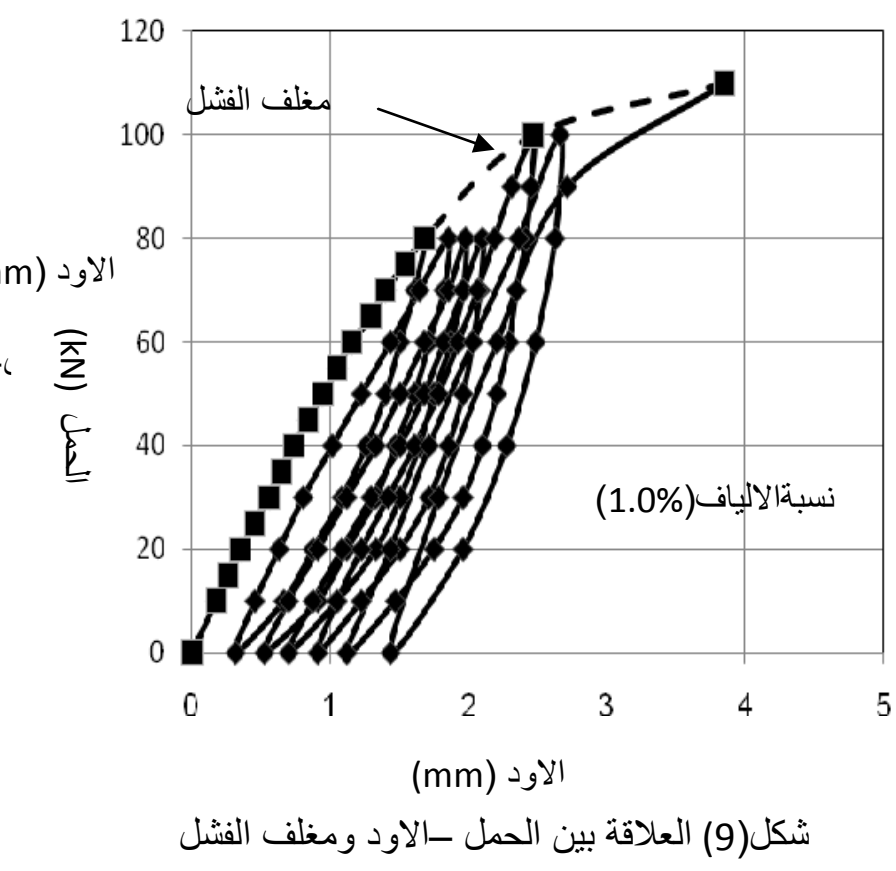

شكل(9) العلاقة بين الحمل -الاود ومغلف الفشل

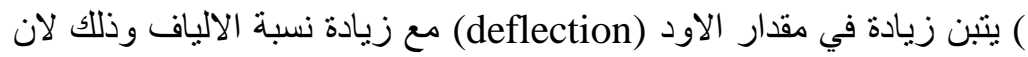

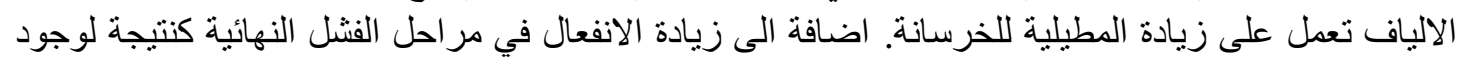

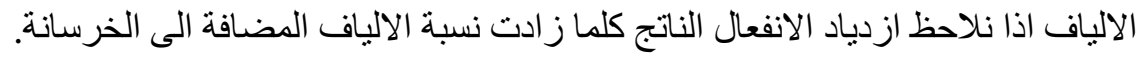

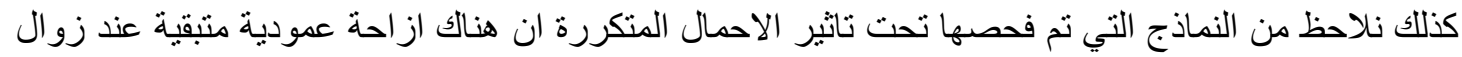

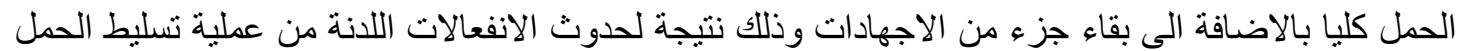
المتكرر ورفحه. 
(10) يمثل العلاقة بين نسبة الالياف مع الاود بثبات الحمل ولدور ات تحميل مختلفة، وتم الاخذ بنظر

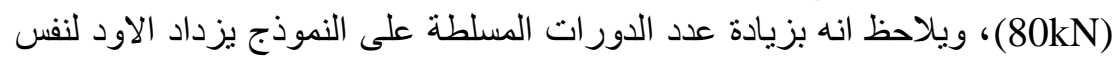

النسبة من الالياف دون ان يكون لهذه الالياف تاثير كبير على مقاومة الانضغاط.

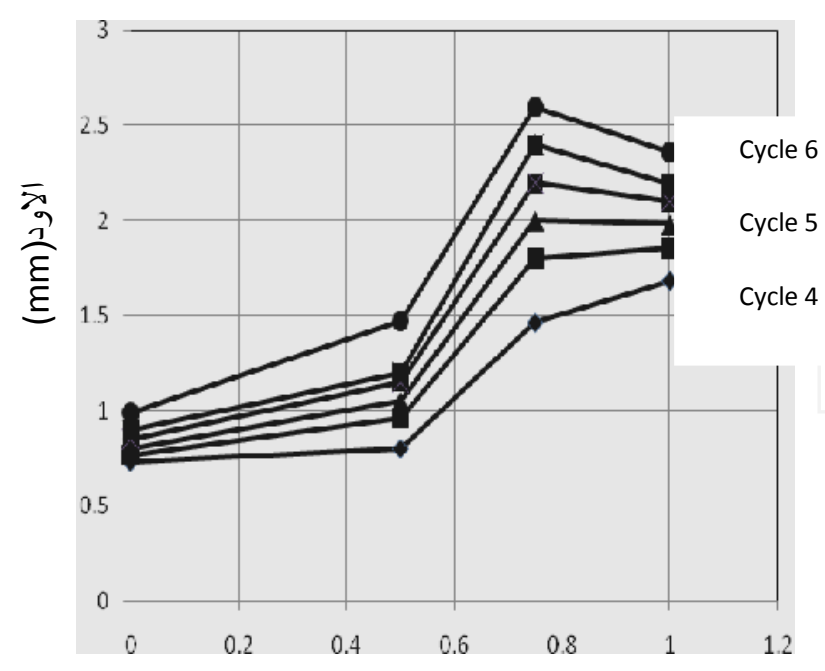

نسبة الالياف( \%)

شكل(10) تأثير عدد الدورات على الاود المتبقي

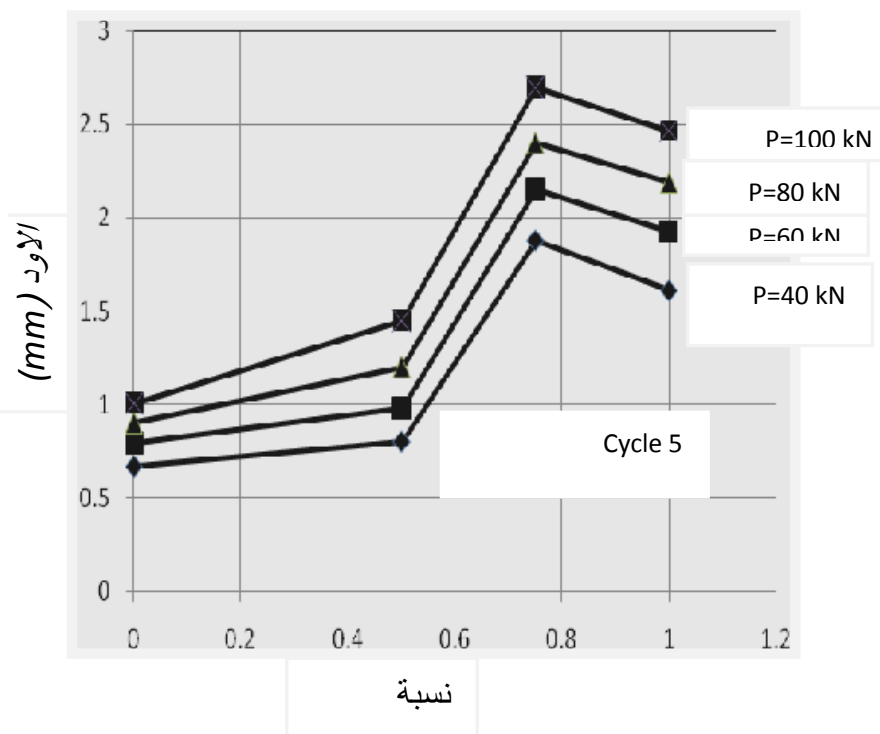

شكل(11) تاثثير الاحمال المتكررة على الاود

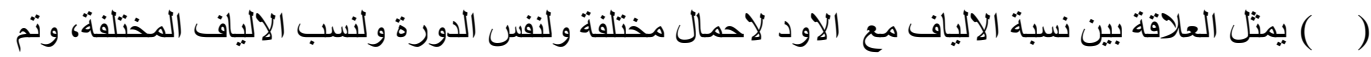

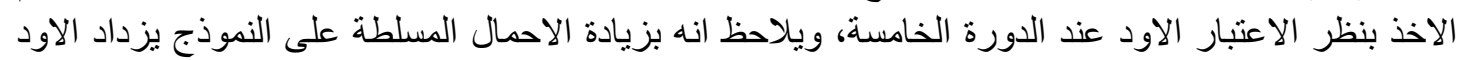

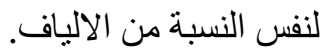

ونتيجة للتحميل المتكرر، يتعرض العضو الخرساني الى نقصان في المطيلية، ويمكن تعريف المطيلية بأنها (Yield)

النسبة بين الاود عند اقصى مقاومة (Ultimate) (12) نلاحظ زيادة المطيلية بزيادة نسبة الالياف، كونها تعمل على زيادة الئة

الاود الناتج خصوصا في مر احل الفنشل النهائية.

نلاحظ ايضا ان المطيلية تزداد كما يزداد الاود وتقل الجساءة وذلك بسبب السحق الذي يحدث في النموذج نتيجة

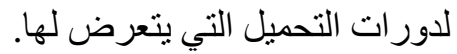

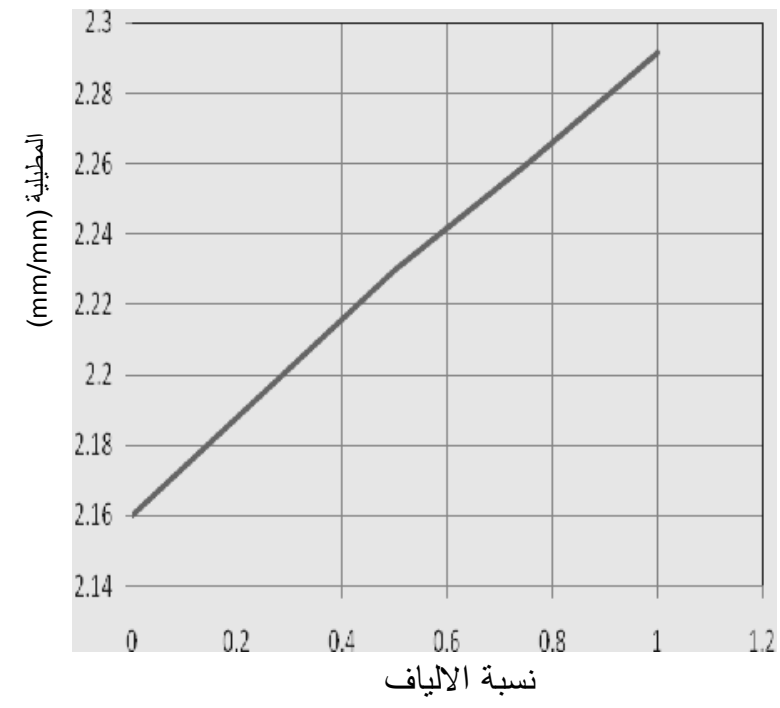

شكل(12)العلاقة بين نسبة الالياف

و المطيلية

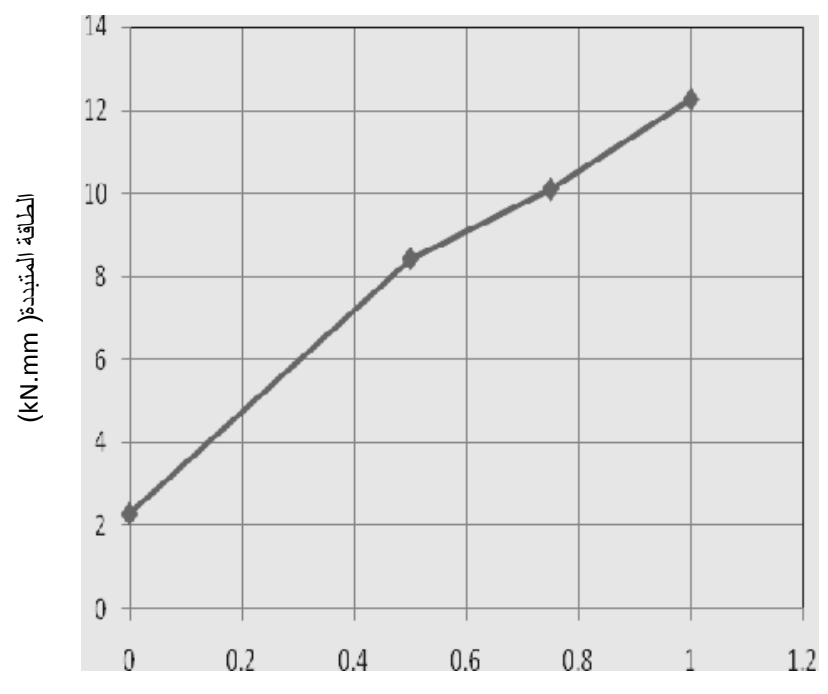

نسبة الالياف (\%)

شكل(13) العلاقة بين نسبة الالياف و الطاقة المتبددة 
عند التحميل ورفع التحميل، لا يتبع المنحني الصاعد نفس مسار المنحني النازل، والفرق بين المنحنيين يمكن تعريفه بأنه مقدار الطاقة المتبددة او المفقودة والناتجة من عملية التحميل ورفع التحميل (ويمكن ملاحظتها في

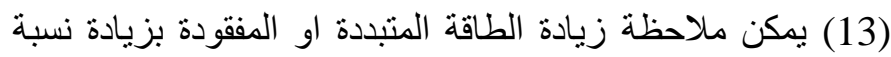
الالياف كون الالياف تعمل على تكوين شقوق متعددة نتيجة لوجودها داخل الخرسانة، مما يؤدي الى تشنتيت كمية لطية

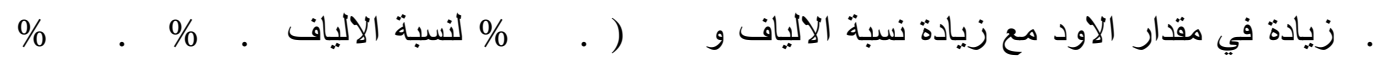

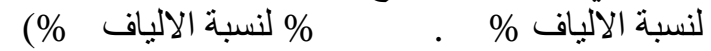
• وجود ازاحة عمودية متبقية عند زوال الحمل كليا بالاضافة الى بقاء جزء من من الاجهادات وذللك نتيجة

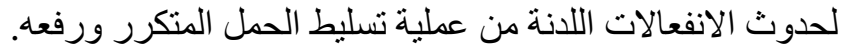

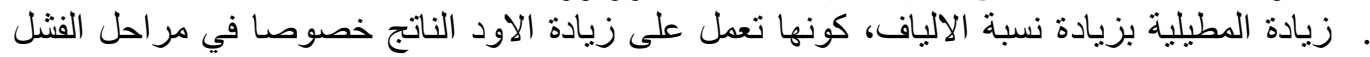

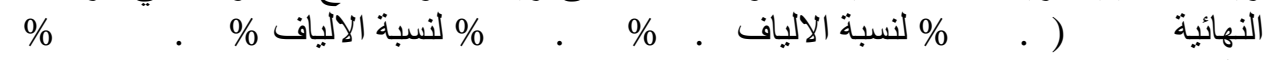
الالياف \% \% الجائ. • تقل الجساءة بزيادة عدد دورات التحميل وذللك بسبب السحق الذي يحدث في النموذج نتيجة لتكرار التحميل ورفعه في كل دورة. 
• زيادة الطاقة المتبددة او المفقودة بزيادة نسبة الالياف كون الالياف تعمل على تكوين شقوق متعددة نتيجة

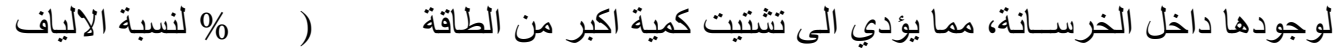

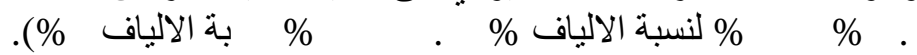

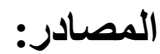

1. Sinha, B.Y and Hsu, Cheng-Tzu, "Stress-Strain Behavior of Concrete under Cyclic loading ",ACI Materials Journal , Vol. .95, No. 2 , March-April 1998,pp.178-193

2. Agrawal,G.L.,Tulinnd Gerstle,K.H,"Response of Doubly Reinforced Concrete Beams to Cyclic Loading ",ACI Journal ,Proceeding, Vol. 66,No.9,sep 1969 ,pp.832-835.

3. Al -Sulayfani, Bayar J.," Contribution A L'etude Comportement Des Ossatures En Beton Arme Sous Solicitations Cycliques Analysis Non-Linear Globale", Docteur De L'Université De Nantes, Sepecialite Genie civil, No.87St, 1986.

4. Thammanoon Denpongpan, "Effect of Reversed Loading on Shear Behavior of Reinforced Concrete", A Dissertation Submitted To Kochi University of Technology In Partial Fulfillment of Requirements For The Degree of Master of Engineering, January, 2001.

5. Hyo - Gyoung Kwak and Sun - Pil Kim, "Nonlinear Analysis of R.C. Beam Subjected to Cyclic Loading", Journal of Structural Engineering,vol.127,No.12, Des. 2001, PP.1436-1444.

6. Synder, M. and Lankard, D.R., "Factors Affecting the Flexural Strength of Steel Fibers Concrete ", ACI Journal, proceeding, Vol.69, No.2, Feb. 1972,pp.96-100.

7. Sakai, M. and, Nakamura ,N., "Analysis of Flexural Behavior of steel fiber Reinforced Concrete "proceeding ,RILEM Symposium RC 86 on Development in fiber Reinforced cement and concrete,Vol.1, RILEM Technical Committee 49-TFR,July 1986 ,pp.27-34.

8. British Standards Institute, B.S:1983"Aggregates from Natural Sources for Concrete". 
9. Shah, R., Mishra,S., "Crack and Deformation Charachteristics of SFRC Deep Beams", IE (1) Journal CV ,Vol. 85 ,May ,2004 ,pp.44-48. 